\title{
Romantic Relationship Dissolution, Microbiota, and Fibers
}

\author{
Jie-Yu Chuang ${ }^{1,2 *}$ \\ ${ }^{1}$ Department of Psychiatry, Cardinal Tien Hospital, New Taipei City, Taiwan, ${ }^{2}$ School of Medicine, College of Medicine, Fu Jen \\ Catholic University, New Taipei City, Taiwan
}

Microbiota inhabit nearly every part of our body with the gut microbiota representing the greatest density and absolute abundance. The gut-microbiota-brain axis facilitates bidirectional communication between gut microbiota and the brain. For instance, romantic relationship not only brings joy, it is also associated with increased gut microbiota diversity and health benefits whereas reduced microbiota diversity is related to obesity, cardiac disease, type 2 diabetes, and inflammatory disorders. Research has shown that dietary fibers may increase microbiota diversity and exert antidepressant effect. Among a plethora of life stressors, romantic relationship dissolution is a relatively common and painful experience that people encounter from time to time.

OPEN ACCESS

Edited by:

Esther Aarts,

Radboud University

Nijmegen, Netherlands

Reviewed by:

Mirjam Bloemendaal,

Radboud University Nijmegen Medical

Centre, Netherlands

Veronica Witte,

Max Planck Institute for Human

Cognitive and Brain

Sciences, Germany

*Correspondence:

Jie-Yu Chuang

xiliwu3@gmail.com

Specialty section:

This article was submitted to

Nutrition and Brain Health,

a section of the journal

Frontiers in Nutrition

Received: 18 January 2021

Accepted: 24 March 2021

Published: 15 April 2021

Citation:

Chuang J-Y (2021) Romantic

Relationship Dissolution, Microbiota, and Fibers. Front. Nutr. 8:655038.

doi: 10.3389/fnut.2021.655038
Depressed mood, social isolation and poor intake are all associated with romantic relationship dissolution. In this article, it is hypothesized that romantic relationship dissolution is accompanied by decreased gut microbiota diversity which could be corrected with the ingestion of dietary fibers with an additional antidepressant benefit.

Keywords: romantic relationship, microbiota, microbiome, love, gut-microbiota-brain axis, fiber, romantic relationship dissolution

\section{GUT-MICROBIOTA-BRAIN AXIS, STRESS AND DIET}

Microbiota inhabit nearly every part of our body with the gut microbiota representing the greatest density and absolute abundance (1). It remains elusive what definitively constitutes an optimal gut microbial profile other than one exhibiting both stability and diversity (2). Microbiota diversity may promote stability and resilience of gut microbiota and is associated with social interaction, infection resistance, improved immunity and reduced inflammation (3). Reduced microbiota diversity is related to obesity, cardiac disease, type 2 diabetes, and inflammatory disorders (4).

The gut-microbiota-brain axis denotes the bidirectional interaction between gut microbiota and brain. Microbiota can produce neurotransmitters (GABA, oxytocin, noradrenaline, dopamine, serotonin, etc.), amino acids (tyramine, tryptophan, etc.) and metabolites (short-chain fatty acids, etc.) which travel through portal circulation to influence immune system, enteric nervous system, gut barrier integrity and the central nervous system (presumably via vagus nerve) (1). Conversely, in response to stress, the hypothalamic-pituitary-adrenal axis can produce cortisol affecting the intestinal barrier integrity, changing the gut environment, and finally altering the gut microbiota composition (1) resulting in a reduction of microbiota diversity (5). For instance, fecal lactic acid bacterial levels are found to be significantly reduced when undergraduate students experience high academic stress (6). Similarly, marital distress has been associated with increased gut permeability (7), possibly changing gut microbiota composition. Moreover, stress might prompt unhealthy food choices, alter metabolic responses to food, and adjust microbiota constitution (8). 
Environmental factors and health behaviors might explain more microbiota variability than do genetic factors (9). Among these environmental factors, diet has emerged as one of the major predictors of gut microbiota composition (8). To confer a health benefit, prebiotic (such as fibers) is dietary supplement which is selectively utilized by microbiota whereas probiotic (such as Lactobacillus) is live microorganism ingested (2). Recently, there has been a surge in studies exploring the beneficial effects of prebiotics and probiotics on mental health (2). Nevertheless, results from these studies are only slowly being translated from animals to humans (10). Unstable results are reported in a recent meta-analysis exploring the efficacy of probiotics on stress reaction with the authors urging more large-scale and welldesigned clinical trials in the future (11). Technical difficulties with probiotics include: ensuring the survival of probiotics to reach and colonize the intestines, and selection of appropriate bacterial strains (2). Intervention with prebiotics can globally improve gut microbiota status in contrast to the use of specific probiotics (2). In sum, an optimal diet therapy mitigating the detrimental effect of stress on gut-microbiota-brain axis remains undetermined.

\section{ROMANTIC RELATIONSHIP DISSOLUTION}

In 1967, Holmes and Rahe developed the Social Readjustment Rating Scale to identify major life stressors. Among the 43 life stressors, romantic relationship dissolution (such as death of spouse or divorce) has been found to be the most traumatic one (12). Nearly $85 \%$ of people experience at least one romantic relationship dissolution in their lifetime (13). This universal experience might lead to grief and depression (14). Romantic relationship dissolution has been referred to as "heartbreak" or "post-relationship grief" (15). Indeed, some even commit suicide following the termination of romantic relationships (16). Romantic relationship dissolution might be accompanied by anxiety, fear, anger, panic, worry, sadness, emotional numbness, loss of purpose, poor concentration, poor memory, poor function, and various somatic symptoms such as loss of appetite and even impaired immunity (15). Copious studies have shown compromised immune function in divorced compared to married adults, such as higher antibody titers to Epstein-Barr virus and lower percentage of natural killer cell activity $(17,18)$. Furthermore, aberrant brain activations in the cerebellum, insula, pre-frontal cortex, anterior temporal cortex, and anterior cingulate after romantic relationship dissolution have been documented (19).

To sum up, romantic relationship dissolution is a universal and excruciating life stressor impacting both mental and physical health. With the emergence of COVID-19 pandemic, a deterioration of romantic relationship has been found by largescale, cross-sectional online questionnaire studies in both US (20) and China (21). Presumably, many people might experience romantic relationship dissolution, and some people might need professional mental help. Nevertheless, due to quarantine and home confinement, opportunities to deliver professional clinical support via direct encounters are greatly curtailed in this crisis
(22). Accordingly, this article introduces a self-help and easilyimplemented hypothetical treatment for romantic relationship dissolution, as described in the following sections.

\section{ROMANTIC RELATIONSHIP DISSOLUTION AND MICROBIOTA}

Couples demonstrate more similar gut microbiota than siblings (4). Therefore, it has been postulated that romantic relationship holds a strong influence on gut microbiota, possibly more powerful than shared genetics and early life environments among siblings (4). The mechanism linking romantic relationship to microbiota remains elusive with some speculations. Firstly, couples might share their microbiota via kissing. The microbiota on the dorsal surface of the tongue has been found to be more similar among partners (Morisita-Horn index $=0.37$ ) than unrelated individuals (Morisita-Horn index $=0.55$ ) (23). Indeed, salivary microbiota is more similar with higher frequency of kissing between couples (23). Secondly, another study shows that transfer of microbiota between couples might occur through shared environment and direct skin contact as cohabiting couples demonstrate similar skin microbiota, especially on their feet, likely reflecting collection of dust (24). Thirdly, behavioral concordance might result in the similarity between partner, such as diet, exercise, sleep, smoking, and alcohol consumption (25). Indeed, diet has a major impact on gut microbiota (26), and dietary patterns play a significant role in the shared gut microbiota in couples (27). Consequently, the above-mentioned evidence might suggest the considerable impact of romantic relationship on gut microbiota.

One study found a significant correlation between larger social network size and increased microbiota diversity (3). It has been speculated that social interaction allows microbiota transmission between subjects and contributes to increased microbiota diversity which counterbalances sociability-related enhanced exposure to infectious agents (5). Indeed, cohabiting spouses, especially those with close relationships, are shown to have more microbiota diversity than those living alone (4). Opposingly, stress has been associated with a reduction of microbiota diversity (5), especially interpersonal stress (conflict, romantic relationship dissolution, etc.) might arise during social interaction and result in a reduction of microbiota diversity (5). Furthermore, it has been demonstrated that depression is also associated with decreased gut microbiota diversity (28). Given that romantic relationship dissolution is frequently associated with solitary living, stressful feeling, and depressive tendency (29), it is speculated that romantic relationship dissolution might be related to decreased microbiota diversity.

In short, studies have suggested that romantic relationship might significantly influence microbiota and is associated with increased microbiota diversity (presumably due to shared diet or microbiota transfer between couples, such as kissing and touching) than singleton. Despite lack of studies directly exploring the interaction between romantic relationship dissolution and microbiota, it is hypothesized that 
romantic relationship dissolution might be related to decreased microbiota diversity.

\section{ROMANTIC RELATIONSHIP DISSOLUTION, MICROBIOTA AND FIBERS: THE HYPOTHESIS}

Diet has a major impact on gut microbiota diversity (26). Dietary fibers are considered as prebiotic, and is associated with increased microbiota diversity (26). Dietary fibers include non-starch polysaccharides, resistant oligosaccharides, lignin, and resistant starch (30). Dietary fiber is defined as edible carbohydrate polymer with three or more monomeric units that are resistant to the endogenous digestive enzymes and neither hydrolyzed nor absorbed in the small intestine (26). Subsequently, gut bacteria can degrade dietary fibers and provide a plethora of simple oligomers serving as an energy source for fermentative microbiota and promote the diversification of gut microbiota (31). Short-chain fatty acids (SCFAs) can then be fermented from fibers by gut microbiota. SCFAs stimulate mucus production, promote generation of colonic regulatory $\mathrm{T}$ cells, increase colonic mineral absorption (26), induce gluconeogenesis, and serve as an energy source for colonocyte (32).

Dietary fiber potentially reduces inflammation (via modifying $\mathrm{pH}$ and the gut permeability) resulting in alteration of the neurotransmitter concentration (30). Indeed, dietary fiber intake has been found to inversely associated with depression $(30,33,34)$. Several potential antidepressant mechanisms of dietary fibers have been speculated: (1) SCFAs produced by gut microbiota fermentation of dietary fibers can inhibit histone deacetylases and increase antidepressant brain-derived neurotrophic factor (BDNF) (2) SCFAs might activate G-proteincoupled receptor and increase antidepressant norepinephrine (3) Fiber intake might potentiate gut microbiota to produce more antidepressant tryptophan, serotonin, and GABG (4) SCFAs can decrease gut permeability with the subsequent decreased serum level of bacteria-produced lipopolysaccharides leading to reduced inflammation whereas inflammation is associated with depression (5) Bicarbonate is released during the production of SCFAs leading to a reduction in intestinal $\mathrm{pH}$ and inhibiting lipopolysaccharides-producing bacteria (30).

In sum, it is hypothesized that romantic relationship dissolution is associated with significantly decreased gut microbiota diversity which might be corrected via dietary fiber ingestion with an additional antidepressant effect.

\section{DISCUSSION}

Romantic relationship dissolution is seldom addressed in scientific literature in spite of its enormous impact to emotional and physical health. This article presents a preliminary hypothesis linking romantic relationship dissolution with gut microbiota and dietary fibers.

To test this hypothesis, several issues should be considered. Firstly, influences of fibers on the gut microbiota has a significant interpersonal variation (35). Some might have problems tolerating high doses of fibers (flatulence, bloating, stomachaches, etc.) (26). Consequently, instead of a one-fits-all high-fiber diet, future studies might focus on designing a personalized highfiber diets for people after romantic relationship dissolution (current dietary recommendation of dietary fibers is around 25-30 g/day (32), higher amount is suggested for romantic relationship dissolution). Secondly, instead of stress-provoking, romantic relationship dissolution can sometimes be stressrelieving and not-applicable for this hypothesis. Thirdly, despite potential biases in participant recruitment and study design, sex differences in emotional responsiveness (36) and resilience (37) to romantic relationship dissolution are suggested. A significant sex difference in depression prevalence is also wellacknowledged (38). Moreover, sex differences have also been found in microbiota diversity (3), degree of microbiota similarity with family members/spouse (39), and effect of fiber ingestion on microbiota (40). Therefore, sex difference in the interaction between romantic relationship dissolution, microbiota, and impact of fibers warrant careful exploration in future studies.

Stress has a great impact on microbiota. Although romantic relationship dissolution stands out as one of the most traumatic life stressors (12), other stressors might also be related to change of dietary habits, lack of physical contacts with others and depressed mood. Consequently, these stressors might influence microbiota to the similar extent as romantic relationship dissolution. For instance, a significant change in microbial environment is expected when one moves out of one's parents' house to live alone in a new city. Similar extent of microbiota alteration can also be found when one is under quarantine in a foreign country and devoid of physical contacts with others. In these situations, fiber supplementation might be able to counteract the effect of stress on gut microbiota diversity. Future studies are warranted to explore the interaction between various life stressors, microbiota, and fibers.

\section{DATA AVAILABILITY STATEMENT}

The original contributions presented in the study are included in the article/supplementary material, further inquiries can be directed to the corresponding author/s.

\section{AUTHOR CONTRIBUTIONS}

J-YC developed the hypothesis and wrote the manuscript.

\section{FUNDING}

J-YC was funded by Cardinal Tien Hospital. 


\section{REFERENCES}

1. Morais LH, Schreiber HL, Mazmanian SK. The gut microbiota-brain axis in behaviour and brain disorders. Nat Rev Microbiol. (2021) 19:24155. doi: 10.1038/s41579-020-00460-0

2. Long-Smith C, O'Riordan KJ, Clarke G, Stanton C, Dinan TG, Cryan JF. Microbiota-gut-brain axis: New therapeutic opportunities. Annu Rev Pharmacol Toxicol. (2020) 60:477502. doi: 10.1146/annurev-pharmtox-010919-023628

3. Johnson KVA. Gut microbiome composition and diversity are related to human personality traits. Hum Microbiome J. (2020) 15:100069. doi: 10.1016/j.humic.2019.100069

4. Dill-McFarland KA, Tang ZZ, Kemis JH, Kerby RL, Chen G, Palloni A, et al. Close social relationships correlate with human gut microbiota composition. Sci Rep. (2019) 9:703. doi: 10.1038/s41598-018-37298-9

5. Sherwin E, Bordenstein SR, Quinn JL, Dinan TG, Cryan JF. Microbiota and the social brain. Science. (2019) 366:eaar2016. doi: 10.1126/science.aar2016

6. Knowles SR, Nelson EA, Palombo EA. Investigating the role of perceived stress on bacterial flora activity and salivary cortisol secretion: a possible mechanism underlying susceptibility to illness. Biol Psychol. (2008) 77:1327. doi: 10.1016/j.biopsycho.2007.09.010

7. Kiecolt-Glaser JK, Wilson SJ, Bailey ML, Andridge R, Peng J, Jaremka LM, et al. Marital distress, depression, and a leaky gut: translocation of bacterial endotoxin as a pathway to inflammation. Psychoneuroendocrinology. (2018) 98:52-60. doi: 10.1016/j.psyneuen.2018.08.007

8. Madison A, Kiecolt-Glaser JK. Stress, depression, diet, and the gut microbiota: human-bacteria interactions at the core of psychoneuroimmunology and nutrition. Curr Opin Behav Sci. (2019) 28:105-10. doi: 10.1016/j.cobeha.2019.01.011

9. Rothschild D, Weissbrod O, Barkan E, Kurilshikov A, Korem T, Zeevi D, et al. Environment dominates over host genetics in shaping human gut microbiota. Nature. (2018) 555:210-5. doi: 10.1038/nature25973

10. Foster JA, Rinaman L, Cryan JF. Stress \& the gut-brain axis: regulation by the microbiome. Neurobiol Stress. (2017) 7:12436. doi: 10.1016/j.ynstr.2017.03.001

11. Zhang N, Zhang Y, Li M, Wang W, Liu Z, Xi C, et al. Efficacy of probiotics on stress in healthy volunteers: a systematic review and meta-analysis based on randomized controlled trials. Brain Behav. (2020) 10:e01699. doi: 10.1002/brb3.1699

12. Holmes TH, Rahe RH. The social readjustment rating scale. J Psychosom Res. (1967) 11:213-8. doi: 10.1016/0022-3999(67)90010-4

13. Battaglia DM, Richard FD, Datteri DL, Lord CG. Breaking up is (relatively) easy to do: a script for the dissolution of close relationships. J Soc Pers Relat. (1998) 15:829-45. doi: 10.1177/0265407598156007

14. Keller MC, Nesse RM. Is low mood an adaptation? Evidence for subtypes with symptoms that match precipitants. J Affect Disord. (2005) 86:2735. doi: 10.1016/j.jad.2004.12.005

15. Morris CE, Reiber C. Frequency, intensity and expression of post-relationship grief. EvoS J. (2011) 3:1-11. Available online at: https://evostudies.org/wpcontent/uploads/2011/01/MorrisVol3Iss1.pdf

16. Love HA, Nalbone DP, Hecker LL, Sweeney KA, Dharnidharka P. Suicidal risk following the termination of romantic relationships. Crisis. (2018) 39:16674. doi: 10.1027/0227-5910/a000484

17. Kiecolt-Glaser JK. Stress, personal relationships, and immune function: health implications. Brain Behav Immun. (1999) 13:61-72. doi: 10.1006/brbi.1999.0552

18. Sbarra DA, Hazan C. Coregulation, dysregulation, self-regulation: an integrative analysis and empirical agenda for understanding adult attachment, separation, loss, and recovery. Personal Soc Psychol Rev. (2008) 12:14167. doi: 10.1177/1088868308315702

19. Najib A, Lorberbaum JP, Kose S, Bohning DE, George MS. Regional brain activity in women grieving a romantic relationship breakup. Am J Psychiatry. (2004) 161:2245-56. doi: 10.1176/appi.ajp.161.12.2245

20. Luetke M, Hensel D, Herbenick D, Rosenberg M. Romantic relationship conflict due to the covid-19 pandemic and changes in intimate and sexual behaviors in a nationally representative sample of american adults. J Sex Marital Ther. (2020) 46:747-62. doi: 10.1080/0092623X.2020.1 810185

21. Li G, Tang D, Song B, Wang C, Qunshan S, Xu C, et al. Impact of the COVID-19 pandemic on partner relationships and sexual and reproductive health: cross-sectional, online survey study. J Med Internet Res. (2020) 22:e20961. doi: 10.2196/20961

22. Pfefferbaum B, North CS. Mental health and the covid-19 pandemic. N Engl J Med. (2020) 383:510-12. doi: 10.1056/NEJMp2008017

23. Kort R, Caspers M, van de Graaf A, van Egmond W, Keijser B, Roeselers G. Shaping the oral microbiota through intimate kissing. Microbiome. (2014) 2:41. doi: 10.1186/2049-2618-2-41

24. Ross AA, Doxey AC, Neufeld JD. The skin microbiome of cohabiting couples. MSystems. (2017) 2:e00043-17. doi: 10.1128/mSystems.00043-17

25. Kiecolt-Glaser JK. Marriage, divorce, and the immune system. Am Psychol. (2018) 73:1098-108. doi: 10.1037/amp0000388

26. Makki K, Deehan EC, Walter J, Bäckhed F. The impact of dietary fiber on gut microbiota in host health and disease. Cell Host Microbe. (2018) 23:705-15. doi: 10.1016/j.chom.2018.05.012

27. Roh Y, Lee J, Kim WG, Yi G, Kim BK, Oh B. Effect of diet change on gut microbiota: observational pilot study of four urban couples. J Obes Metab Syndr. (2017) 26:257-65. doi: 10.7570/jomes.2017.26.4.257

28. Kelly JR, Borre Y, O' Brien C, Patterson E, El Aidy S, Deane J, et al. Transferring the blues: depression-associated gut microbiota induces neurobehavioural changes in the rat. J Psychiatr Res. (2016) 82:10918. doi: 10.1016/j.jpsychires.2016.07.019

29. Field T, Diego M, Pelaez M, Deeds O, Delgado J. Breakup distress in university students. Adolescence. (2009). 44:705-27. doi: 10.1037/t66170-000

30. Swann OG, Kilpatrick M, Breslin M, Oddy WH. Dietary fiber and its associations with depression and inflammation. Nutr Rev. (2020) 78:394411. doi: 10.1093/nutrit/nuz072

31. Doré J, Blottière $H$. The influence of diet on the gut microbiota and its consequences for health. Curr Opin Biotechnol. (2015) 32:1959. doi: 10.1016/j.copbio.2015.01.002

32. Sanz Y, Romaní-Perez M, Benítez-Páez A, Portune KJ, Brigidi P, Rampelli S, et al. Towards microbiome-informed dietary recommendations for promoting metabolic and mental health: opinion papers of the mynewgut project. Clin Nutr. (2018) 37 (6 Pt. A):2191-7. doi: 10.1016/j.clnu.2018.07.007

33. Miki T, Eguchi M, Kurotani K, Kochi T, Kuwahara K, Ito R, et al. Dietary fiber intake and depressive symptoms in Japanese employees: the furukawa nutrition and health study. Nutrition. (2016) 32:5849. doi: 10.1016/j.nut.2015.11.014

34. Ramin S, Mysz MA, Meyer K, Capistrant B, Lazovich DA, Prizment A. A prospective analysis of dietary fiber intake and mental health quality of life in the iowa women's health study. Maturitas. (2020) 131:17. doi: 10.1016/j.maturitas.2019.10.007

35. Kolodziejczyk AA, Zheng D, Elinav E. Diet-microbiota interactions and personalized nutrition. Nat Rev Microbiol. (2019) 17:74253. doi: 10.1038/s41579-019-0256-8

36. Morris CE, Reiber C, Roman E. Quantitative sex differences in response to the dissolution of a romantic relationship. Evol Behav Sci. (2015) 9:27082. doi: 10.1037/ebs0000054

37. DeLecce T, Weisfeld G. An evolutionary explanation for sex differences in nonmarital breakup experiences. Adapt Hum Behav Physiol. (2016) 2:23451. doi: 10.1007/s40750-015-0039-Z

38. Chuang J-Y, Hagan CC, Murray GK, Graham JME, Ooi C, Tait R, et al. Adolescent major depressive disorder: neuroimaging evidence of sex difference during an affective go/no-go task. Front Psychiatry. (2017) 8:119. doi: 10.3389/fpsyt.2017.00119

39. Brito IL, Gurry T, Zhao S, Huang K, Young SK, Shea TP, et al. Transmission of human-associated microbiota along family and social networks. Nat Microbiol. (2019) 4:964-71. doi: 10.1038/s41564-019-0409-6

40. Kim YS, Unno T, Kim BY, Park MS. Sex differences in gut microbiota. World J Mens Health. (2020) 38:48-60. doi: 10.5534/wjmh.190009

Conflict of Interest: The author declares that the research was conducted in the absence of any commercial or financial relationships that could be construed as a potential conflict of interest.

Copyright (C) 2021 Chuang. This is an open-access article distributed under the terms of the Creative Commons Attribution License (CC BY). The use, distribution or reproduction in other forums is permitted, provided the original author(s) and the copyright owner(s) are credited and that the original publication in this journal is cited, in accordance with accepted academic practice. No use, distribution or reproduction is permitted which does not comply with these terms. 